\title{
Identifying New Molecules from Comparison of Herschel-HIFI Spectra with $a b$ initio Computational Spectra
}

\author{
Naseem Rangwala ${ }^{1,2}$, Xinchuan Huang ${ }^{1,3}$, Sean Colgan ${ }^{1}$, \\ Timothy Lee ${ }^{1}$ and Ryan Fortenberry ${ }^{4}$ \\ ${ }^{1}$ NASA Ames Research Center mail: naseem.rangwala@nasa.gov, \\ ${ }^{2}$ NASA Postdoctoral Position (Oak Ridge Associated Universities), ${ }^{3}$ The SETI Institute, \\ ${ }^{4}$ Georgia Southern University
}

\begin{abstract}
We will identify new molecules in the interstellar medium by comparing a catalog of theoretical spectra generated by the NASA Ames quantum chemistry group with recent highresolution astronomical line surveys.
\end{abstract}

Keywords. ISM: molecules, astronomical data bases: surveys

A significant fraction of those lines that remain unidentified in recent astronomical line surveys from radio and millimeter telescopes (e.g., Herschel Space Observatory, Green Bank Telescope (GBT), and Atacama Large Millimeter Array (ALMA), e.g. Fig.1), most likely arise from previously undetected molecules. A large repository of reference data (wavelengths and relative intensities) is required to identify new molecules. For many molecules, experimental reference data do not exist. In these cases, quantum chemical computations can provide estimates for these parameters without some of the limitations inherent in generating experimental reference data. Our project will use computational spectra generated by the quantum chemistry group at NASA Ames research center (e.g. Fig.1) to identify new molecules by comparison with existing astronomical line surveys. This will enhance the chemical inventory for Galactic star forming regions, allowing astrochemists to establish or confirm a variety of chemical networks, understand organic chemistry associated with star formation, and inform the studies that investigate the supply pathways of key organic molecules in Earth-like planet formation.
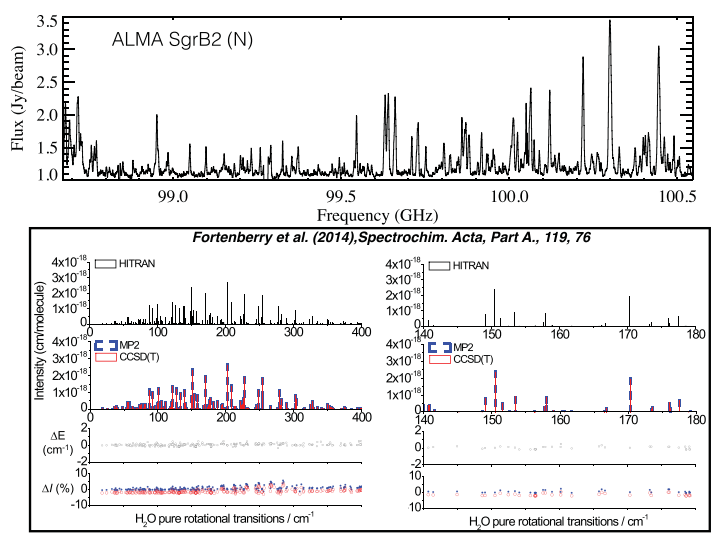

Figure 1. (Top) A portion of data from of SgrB2(N) from the ALMA public archive shows very high density of lines and many are expected to be unidentified. (Bottom) Comparison between the computational data generated by the NASA Ames group and the HITRAN database. Left: Comparison for the rotational transitions of $\mathrm{H} 2 \mathrm{O}$. Right: Closeup view $\left(140-180 \mathrm{~cm}^{-1} ; 1 \mathrm{GHz}\right.$ $\sim 30 \mathrm{~cm}^{-1}$ ). HITRAN spectrum is shown in Black. The computed transitions are directly underneath; blue and red using two different methods. The differences in the line positions and intensities between computations and HITRAN are shown in the 3rd and 4th plots, respectively. 